Check for updates

Cite this: Chem. Commun., 2019,

55,6607

Received 18th April 2019,

Accepted 15th May 2019

DOI: $10.1039 / \mathrm{c} 9 \mathrm{cc} 03032 \mathrm{~h}$

rsc.li/chemcomm

\section{Near-infrared circularly polarized luminescence from chiral Yb(III)-diketonates $\dagger$}

\author{
Francesco Zinna, (D) * Lorenzo Arrico and Lorenzo Di Bari (D) *
}

We report a rare example of near infrared circularly polarized luminescence (NIR-CPL) in the 920-1050 nm region associated with the $f-f$ transitions of $\mathrm{Yb}$ diketonate complexes bearing chiral ancillary ligands. Developing NIR-CPL emitters may have implications from CPL-based (bio)-assays to chiral optoelectronics.

Circularly polarized luminescence (CPL) is a flourishing field gaining more and more interest thanks to its versatility and wide scope. For example, it is successfully employed in (bio)-assays ${ }^{1,2}$ where a luminescent reporter interacts with a (bio)-analyte, triggering or changing its circularly polarized emission. ${ }^{3-5}$ This technique provides advantages in terms of selectivity over non-polarized counterparts in tracking interactions with chiral environments. Moreover, CPL-emitting materials are finding applications in chiral electronics, such as in circularly polarized OLEDs. ${ }^{6-10}$

Extending and consolidating circularly polarized emission in the near infrared (NIR) region would be highly beneficial in order to increase the versatility and applicability of such a technique. Indeed, tissues are relatively transparent to NIR light, due to the weak absorption of skin, blood and water and reduced scattering in such a wavelength region. In particular, an optimal optical window for such a purpose ranges from 650 to $1200 \mathrm{~nm}$, making Yb-centred emission (900-1050 nm) particularly suitable in this context. ${ }^{11,12}$ Moreover, Yb luminescence joins the usual benefits associated with $\mathrm{f}-\mathrm{f}$ lanthanide emission, such as large energy difference between excitation and emission, narrow emission bands and long emission lifetimes. ${ }^{13}$

So far NIR-CPL was only described in pioneering works of Parker et al. on chiral $\mathrm{Yb}$ and Nd complexes based on 1,4,7,10tetraazacyclododecane (cyclen) under UV irradiation. ${ }^{14-16}$ Since then, no further examples of NIR-CPL have been reported. This is probably due to the generally weak emission so far attained in such a region combined with the lack of suitable

Dipartimento di Chimica e Chimica Industriale, Università di Pisa, via G. Moruzzi 13, 56124, Pisa, Italy.E-mail: francesco.zinna@unipi.it, lorenzo.dibari@unipi.it $\dagger$ Electronic supplementary information (ESI) available: Preparation of compounds, NIR-CPL setup description and additional spectra. See DOI: 10.1039/c9cc03032h apparatus for reliable NIR-CPL detection, which makes the measurement of NIR-CPL a difficult task.

Here, we committed to obtaining Yb-based NIR circularly polarized emitters, which could afford a good trade-off between the total luminescence and circular polarization. We took advantage of $\mathrm{Yb}(\mathrm{TTA})_{3}$ (TTA $=$ 2-thenoyltrifluoroacetonate) complexes which are known to show relatively good quantum yields ${ }^{17-19}$ under mild UV irradiation. The coordination sphere of such tris diketonate lanthanide complexes can be easily completed by using chiral neutral ancillary ligands, such as PyBox (pyridine bisoxazoline). ${ }^{20-22}$ This strategy permits high tunability and modularity and allows one to tune independently the antenna (diketonate) and the chiral inducer (ancillary ligand). Therefore, we prepared both the enantiomers of $\mathrm{Yb}(\mathrm{TTA})_{3}{ }^{\mathrm{i}} \mathrm{PrPyBox}$ and $\mathrm{Yb}(\mathrm{TTA})_{3^{-}}$ PhPyBox (Scheme 1) and we studied their NIR-CPL properties.

In order to measure NIR-CPL, we modified our spectrofluoropolarimeter apparatus, ${ }^{23}$ as described in the ESI, $\dagger$ by using a Ag-O-Cs photomultiplier tube as the detector, while polarization discrimination was performed using a photoelastic modulator coupled with an uncoated Glan-Thompson polarizer (Fig. S1, ESI $\dagger$ ). Excitation was performed using a general purpose $365 \mathrm{~nm}$ LED source. With this setup configuration, the total duration for CPL data acquisition was around 9 min for each sample.

We started by assessing whether the compounds gave an intense enough total NIR emission signal (Fig. 1 and 2, bottom).

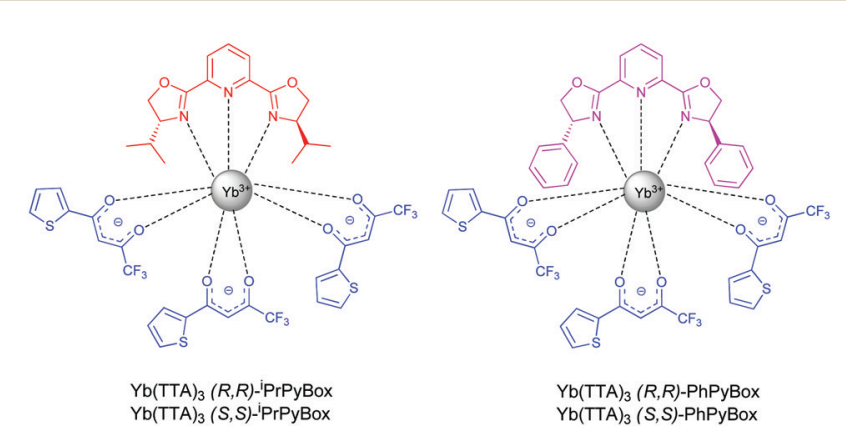

Scheme 1 Compounds investigated in his work. 

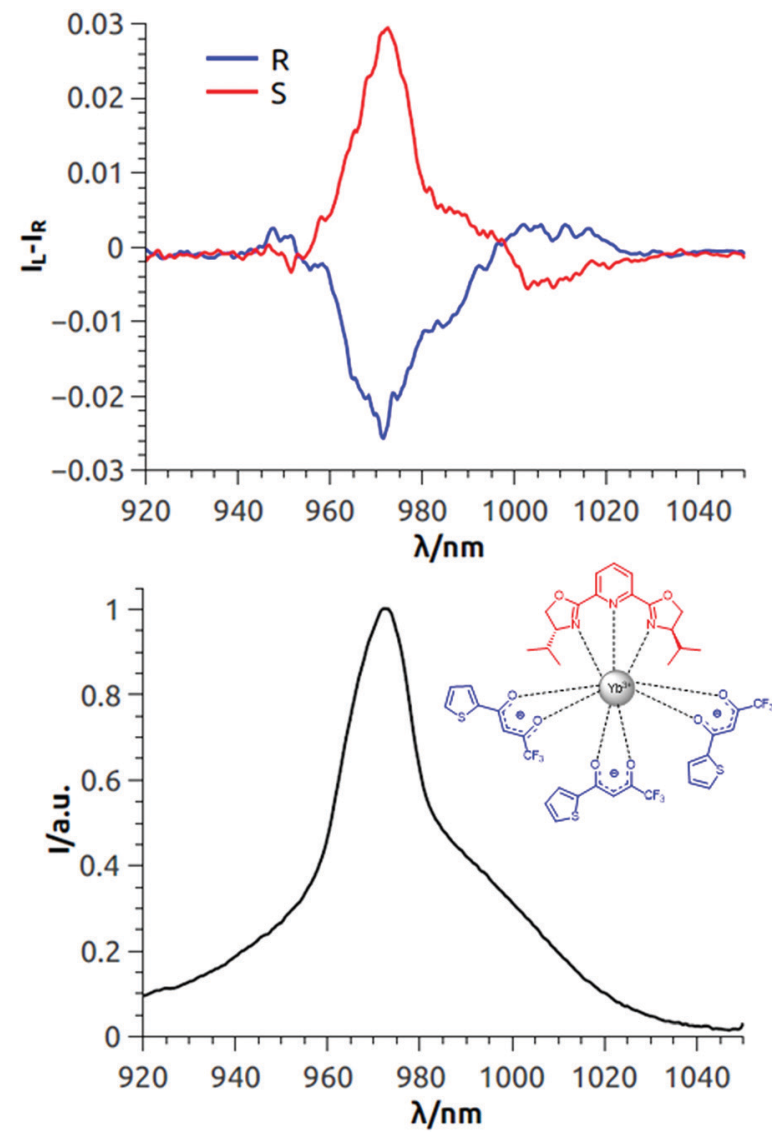

Fig. 1 Top: NIR-CPL spectra of both the enantiomers of $\mathrm{Yb}(\mathrm{TTA})_{3}{ }^{\text {PPrPybox; }}$ bottom: total NIR emission. The spectra are measured in $6 \mathrm{mM} \mathrm{CH}_{2} \mathrm{Cl}_{2}$ solution under $365 \mathrm{~nm}$ irradiation (room temperature).

The quantum yields $\left(Q_{\mathrm{Yb}}^{\mathrm{L}}\right)$ were determined to be $0.69 \%$ and $0.62 \%$ for $\mathrm{Yb}(\mathrm{TTA})_{3}{ }_{3}^{\mathrm{i}}$ PrPyBox and $\mathrm{Yb}(\mathrm{TTA})_{3} \mathrm{PhPyBox}$, respectively, relative to $\mathrm{Yb}(\mathrm{TTA})_{3}\left(\mathrm{H}_{2} \mathrm{O}\right)_{2}\left(Q_{\mathrm{Yb}}^{\mathrm{L}}=0.35 \%\right.$ in toluene $) .{ }^{17}$ Then we investigated the CPL in the $900-1050 \mathrm{~nm}$ region of $\mathrm{Yb}(\mathrm{TTA}){ }_{3}{ }^{\mathrm{i}} \mathrm{PrPyBox}$ in $6 \mathrm{mM} \mathrm{CH}_{2} \mathrm{Cl}_{2}$ solution. A manifold associated with ${ }^{2} \mathrm{~F}_{5 / 2} \rightarrow{ }^{2} \mathrm{~F}_{7 / 2}$ transitions, consisting of both positive and negative bands, was observed (Fig. 1, top). The main feature in the CPL spectrum was the relatively sharp band around $972 \mathrm{~nm}$ with a $g_{\text {lum }}$ factor of -0.025 and +0.029 for $(R, R)$ and $(S, S)$ enantiomers respectively (see also Fig. S2, ESI $\dagger$ ), corresponding to $1^{\prime} \rightarrow 1$ transition (primed and unprimed numbers refer to ${ }^{2} \mathrm{~F}_{5 / 2}$ and to ${ }^{2} \mathrm{~F}_{7 / 2}$ states respectively). This transition is clearly visible in the total luminescence as well (Fig. 1, bottom). Other transitions, associated with $1^{\prime} \rightarrow 2,3,4$ are visible at longer wavelengths. Importantly mirror image spectra were obtained for the two enantiomers ensuring that the spectra were not significantly affected by artifacts due to linear anisotropies in the detection apparatus.

In the case of the NIR-CPL of $\mathrm{Yb}(\mathrm{TTA})_{3}$ PhPyBox $(6 \mathrm{mM}$ $\mathrm{CH}_{2} \mathrm{Cl}_{2}$ solution), a manifold was again observed with $g_{\text {lum }}$ of +0.016 and -0.019 (for $(R, R)$ and $(S, S)$ respectively) at $970 \mathrm{~nm}$ for $1^{\prime} \rightarrow 1$ transition (Fig. 2, top and Fig. S3, ESI $\dagger$ ). In this case, however, such a main band has an opposite sign with respect to the same band observed for $\mathrm{Yb}(\mathrm{TTA})_{3}{ }^{\mathrm{i}} \operatorname{PrPyB}$ ox with the same given absolute configuration of the PyBox unit (i.e., positive for
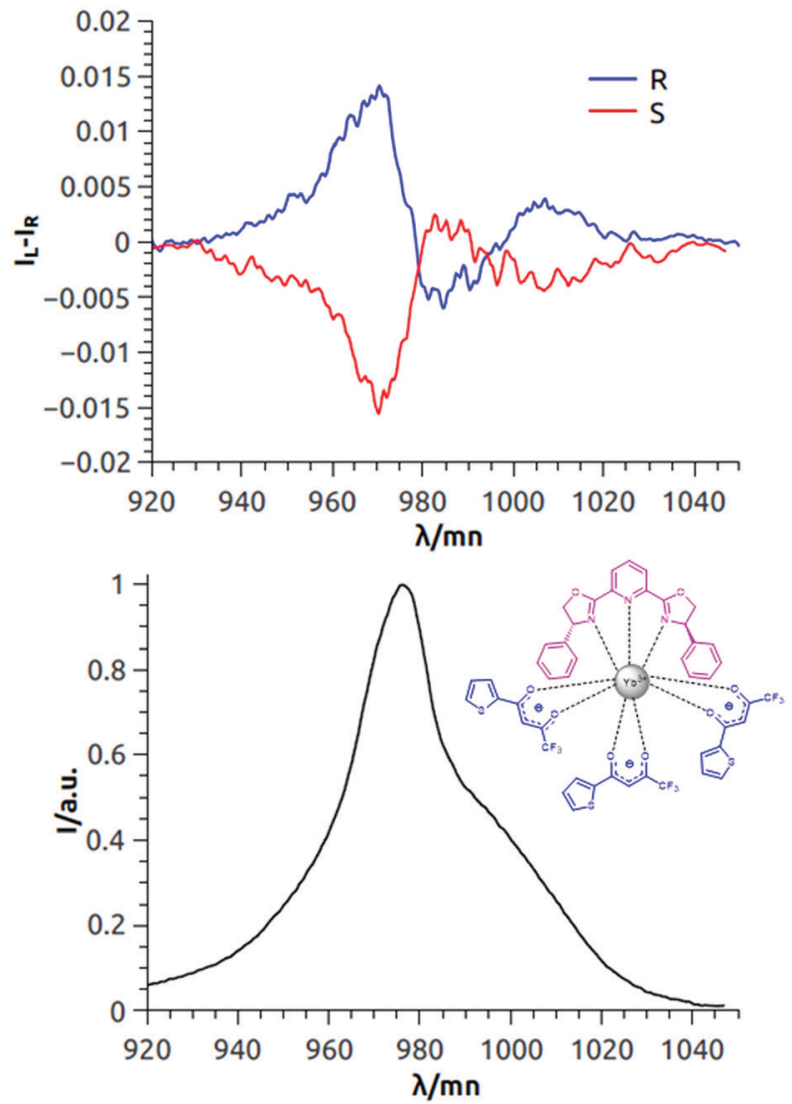

Fig. 2 Top: NIR-CPL spectra of both the enantiomers of $\mathrm{Yb}(T \mathrm{TA})_{3} \mathrm{PhPybox}$; bottom: total NIR emission. The spectra are measured in $6 \mathrm{mM} \mathrm{CH}_{2} \mathrm{Cl}_{2}$ solution under $365 \mathrm{~nm}$ irradiation (room temperature).

$\mathrm{Yb}(\mathrm{TTA})_{3}(S, S)-{ }^{\mathrm{i}}$ PrPyBox and negative for $\mathrm{Yb}(\mathrm{TTA})_{3}(S, S)$-PhPyBox). This inversion is observed also in ECD and NIR-CD spectra (vide infra) and it is in line with what was previously reported for the chiroptical properties of analogous or similar $\mathrm{Sm}, \mathrm{Eu}$ or $\mathrm{Tb}$ complexes..$^{20,22}$

For both the compounds, the overall integral of the NIR-CPL spectra does not vanish, featuring a significant imbalance of the area of one sign over the other $\left(85: 15\right.$ and $90: 10$ for $\mathrm{Yb}(\mathrm{TTA}){ }_{3}{ }_{3}{ }^{\mathrm{PrPyB}}$ Pox and $\mathrm{Yb}(\mathrm{TTA})_{3} \mathrm{PhPybox}$ respectively). This apparent violation of the sum rule can be rationalized by taking into account the fact that these $\mathrm{f}-\mathrm{f}$ transitions gain rotational strength by coupling with the induced electric dipole transition moment of the $\pi-\pi^{*}$ transition on the diketonate moieties (dynamic coupling). ${ }^{2,24,25}$ Therefore, such transitions can no longer be considered isolated and the sum rule does not necessarily apply.

To complete the investigation, ECD studies in the UV and NIR regions were undertaken.

The ECD spectra of all the compounds in the UV domain showed relatively intense Cotton effects at wavelengths lower than $400 \mathrm{~nm}$. With both the compounds, an exciton couplet band allied with the $\pi-\pi^{*}$ transition of the diketonate ligands (absorption band around $344 \mathrm{~nm}$ ) was observed (Fig. S4 and S5, ESI $\dagger$ ). The couplet centre is blue-shifted in the case of $\mathrm{Yb}(\mathrm{TTA}){ }_{3}^{\mathrm{i}} \mathrm{PrPyBox}$ with respect to $\mathrm{Yb}(\mathrm{TTA})_{3} \mathrm{PhPyBox}$, reflecting a different skew angle between the diketonates in the two complexes. Moreover, the sign 

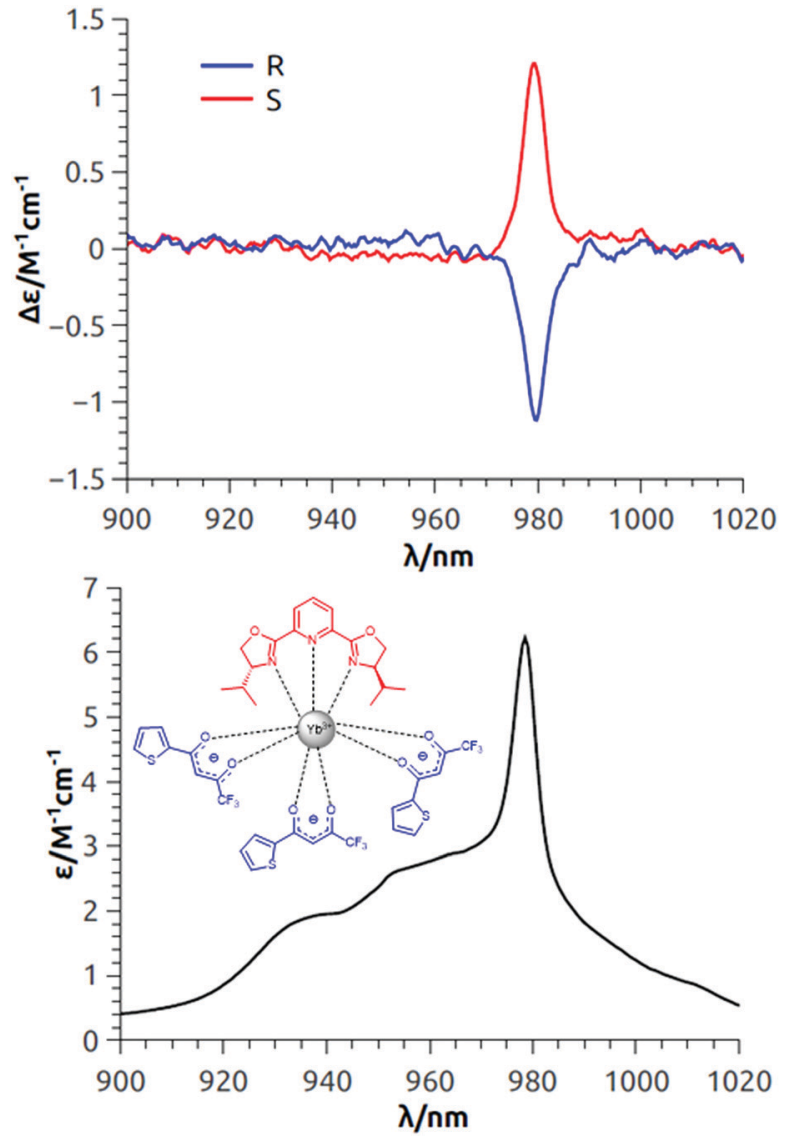

Fig. 3 Top: NIR-ECD spectra of both the enantiomers of $\mathrm{Yb}(T \mathrm{TA}) \mathrm{z}_{3}{ }^{\mathrm{P} P r P y b o x}$;

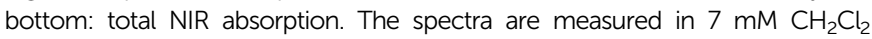
solution (room temperature).

of the couplet is inverted for the two compounds, indicating that the arrangement of the ligands around the metal centre has an opposite handedness in the two compounds, given the same absolute configuration of the PyBox ancillary ligand. This is consistent with the results observed in CPL and in previous reports. ${ }^{20,22}$

Both enantiomeric pairs of the two complexes showed ECD signals associated with ${ }^{2} \mathrm{~F}_{7 / 2} \rightarrow{ }^{2} \mathrm{~F}_{5 / 2}$ transitions in the 900$1020 \mathrm{~nm}$ region. As in the emission/CPL spectrum, the $1 \rightarrow 1^{\prime}$ band around $975 \mathrm{~nm}$ is the main feature of the absorption/ECD spectrum (Fig. 3 and Fig. S6, ESI $\dagger$ ), ${ }^{26}$ especially in the case of $\mathrm{Yb}$ (TTA) ${ }_{3}{ }^{\mathrm{i}}$ PrPyBox. For all the compounds, this transition matches qualitatively (in terms of sign) in both ECD and CPL spectra, as it is expected since it involves the same electronic levels $\left(1 / 1^{\prime}\right)$. However, $g_{\text {abs }}$ values were found to be higher than the corresponding $g_{\text {lum }}$. This can be rationalized by taking into account the fact that for very close transitions with possible different sign, a quantitative comparison between emission and absorption dissymmetry factors is not straightforward. ${ }^{15}$

In conclusion, we have shown that NIR-CPL can be effectively measured on tailored but relatively simple and flexible complexes, taking advantage of $\mathrm{Yb}$ emission. This work falls within the exciting context of circularly polarized emission, contributing a so far extremely rare example of NIR-CPL.
This goes towards the direction of developing new NIR-CPL emitters for applications ranging from NIR-CPL (bio)-assays to chiral NIR optoelectronics.

We thank Prof. Francesco Pineider for assistance with the NIR-CPL setup and Prof. Andrea Pucci and Dr Pierpaolo Minei for kind help with NIR absorption measurements. Financial support from the University of Pisa (PRA 2018_23) is gratefully acknowledged.

\section{Conflicts of interest}

There are no conflicts to declare.

\section{Notes and references}

$\ddagger$ Here we refer to NIR-centred CPL, as opposite to CPL allied with redcentred emission with low energy tails possibly stretching beyond the visible boundaries.

1 R. Carr, N. H. Evans and D. Parker, Chem. Soc. Rev., 2012, 41, 7673-7686.

2 F. Zinna and L. Di Bari, Chirality, 2015, 27, 1-13.

3 J. Yuasa, T. Ohno, H. Tsumatori, R. Shiba, H. Kamikubo, M. Kataoka, Y. Hasegawa and T. Kawai, Chem. Commun., 2013, 49, 4604-4606.

4 S. Orsini, F. Zinna, T. Biver, L. Di Bari and I. Bonaduce, RSC Adv., 2016, 6, 96176-96181.

5 M. Leonzio, A. Melchior, G. Faura, M. Tolazzi, M. Bettinelli, F. Zinna, L. Arrico, L. Di Bari and F. Piccinelli, New J. Chem., 2018, 42, 7931-7939.

6 J. R. Brandt, F. Salerno and M. J. Fuchter, Nat. Rev. Chem., 2017, 1, 0045.

7 Y. Yang, R. C. da Costa, D. M. Smilgies, A. J. Campbell and M. J. Fuchter, Adv. Mater., 2013, 25, 2624-2628.

8 F. Zinna, U. Giovanella and L. Di Bari, Adv. Mater., 2015, 27, 1791-1795.

9 J. R. Brandt, X. Wang, Y. Yang, A. J. Campbell and M. J. Fuchter, J. Am. Chem. Soc., 2016, 138, 9743-9746.

10 F. Zinna, M. Pasini, F. Galeotti, C. Botta, L. Di Bari and U. Giovanella, Adv. Funct. Mater., 2017, 27, 1603719.

11 J.-C. G. Bünzli and S. V. Eliseeva, J. Rare Earths, 2010, 28, 824-842.

12 S. Comby, D. Imbert, A.-S. Chauvin and J.-C. G. Bünzli, Inorg. Chem., 2006, 45, 732-743.

13 J.-C. G. Buenzli and S. V. Eliseeva, Chem. Sci., 2013, 4, 1939-1949.

14 C. L. Maupin, D. Parker, J. G. Williams and J. P. Riehl, J. Am. Chem. Soc., 1998, 120, 10563-10564.

15 C. L. Maupin, R. S. Dickins, L. G. Govenlock, C. E. Mathieu, D. Parker, J. G. Williams and J. P. Riehl, J. Phys. Chem. A, 2000, 104, 6709-6717.

16 R. S. Dickins, J. A. Howard, C. L. Maupin, J. M. Moloney, D. Parker, J. P. Riehl, G. Siligardi and J. G. Williams, Chem. - Eur. J., 1999, 5, 1095-1105.

17 S. Meshkova, Z. Topilova, D. Bolshoy, S. Beltyukova, M. Tsvirko and V. Venchikov, Acta Phys. Pol., A, 1999, 6, 983-990.

18 Z. Ahmed, R. E. Aderne, J. Kai, J. A. Resende and M. Cremona, Polyhedron, 2016, 117, 518-525.

19 B. Li, H. Li, P. Chen, W. Sun, C. Wang, T. Gao and P. Yan, Phys. Chem. Chem. Phys., 2015, 17, 30510-30517.

20 J. Yuasa, T. Ohno, K. Miyata, H. Tsumatori, Y. Hasegawa and T. Kawai, J. Am. Chem. Soc., 2011, 133, 9892-9902.

21 L. Armelao, D. B. Dell'Amico, L. Bellucci, G. Bottaro, L. Di Bari, L. Labella, F. Marchetti, S. Samaritani and F. Zinna, Inorg. Chem., 2017, 56, 7010-7018.

22 M. Górecki, L. Carpita, L. Arrico, F. Zinna and L. Di Bari, Dalton Trans., 2018, 47, 7166-7177.

23 F. Zinna, T. Bruhn, C. A. Guido, J. Ahrens, M. Bröring, L. Di Bari and G. Pescitelli, Chem. - Eur. J., 2016, 22, 16089-16098.

24 S. Di Pietro and L. Di Bari, Inorg. Chem., 2012, 51, 12007-12014.

25 R. Berardozzi and L. Di Bari, ChemPhysChem, 2015, 16, 2868-2875.

26 L. Di Bari, G. Pintacuda and P. Salvadori, J. Am. Chem. Soc., 2000, 122, 5557-5562. 